\title{
Sfrp5/Wnt Pathway: A Protective Regulatory System in Atherosclerotic Cardiovascular Disease
}

\author{
Shan Tong, ${ }^{1,2, *}$ Qingwei Ji, ${ }^{3, *}$ Yu Du, ${ }^{1}$ Xiaogang Zhu, ${ }^{1}$ Caizhong Zhu, ${ }^{2}$ and Yujie Zhou ${ }^{1}$
}

Adipose tissue stores energy and is the largest endocrine organ in the body, producing several adipokines. However, among these adipokines, few play a role in the positive metabolism that promotes good health. Secreted frizzled-related protein (Sfrp)-5, an antagonist that directly binds to Wnt, has attracted interest due to its favorable effects on atherosclerotic cardiovascular disease (ASCVD). This review focuses on Sfrp5 biology and the roles of the Sfrp5/Wnt system in ASCVD.

Keywords: atherosclerotic cardiovascular disease, adipokines, Sfrp5, Wnt signaling pathway

\section{Background}

$\mathrm{C}$ ARDIOVASCULAR DISEASES (CVD) ARE a growing global health problem that results in increased health care burden and decreased life expectancy. Atherosclerotic cardiovascular disease (ASCVD) is the most common cardiovascular disease (CVD). The basic pathological changes during ASCVD include atherosclerosis (AS), obesity, insulin resistance, lipid metabolism disorders, and chronic inflammation. An increasing number of researchers have reported that adipose tissue stores energy and is the largest endocrine organ in the body. Adipose tissue, especially visceral fat, can secrete a variety of fat factors (adipokines), such as leptin, adiponectin, resistin, tumor necrosis factor $(\mathrm{TNF}-\alpha)$, interleukin (IL)-6, visfatin, omentin, retinol binding protein, and secreted frizzled-related protein (Sfrp). These factors may alter the metabolism and functions of cardiac cells, endothelial cells, arterial smooth muscle cells, and inflammatory cells, leading to the development of AS and CVD.

However, few of these factors play a positive role in the metabolism, promoting good health. Sfrp5, a member of the Sfrps family, has been recently identified as a novel adipocytokine that belongs to the category of "good" adipokines (Ouchi and others 2010). Plasma Sfrp5 levels are significantly decreased in patients with obesity, insulin resistance, and related diseases, such as diabetes, AS, and CVD. Recent studies showed that Wnt signaling plays a major role in the progression of heart disease (Gay and Towler 2017) and Sfrp5 acts primarily by inhibiting the Wnt signaling pathway, thereby suppressing the development of AS and CVD (Jaikanth and others 2017). This review focuses on specific aspects of Sfrp5s physiological and pathological functions mediated via the Wnt pathway in the cardiovascular system.

\section{Sfrp5 Structure, Distribution, and Biology}

\section{Sfrps and Wnt signaling pathways}

The Wnt signaling pathway is closely associated with the process of cell proliferation and differentiation. This pathway not only participates in human embryonic development and organ formation but also plays a crucial role in some pathophysiological processes, such as tumor formation, obesity, insulin resistance, fibrosis, and inflammatory responses (Hermans and Blankesteijn 2015; Gay and Towler 2017; Liu and others 2018; Yin and others 2018). Wnt signaling pathway consists of the Wnt protein family, cell membrane receptor frizzled (frizzled, Fz), cytoplasmic

\footnotetext{
${ }^{1}$ Beijing Key Laboratory of Precision Medicine of Coronary Atherosclerotic Disease, Department of Cardiology, 12th ward, Beijing Anzhen Hospital, Beijing Institute of Heart Lung and Blood Vessel Disease, Clinical Center for Coronary Heart Disease, Capital Medical University, Beijing, China.

${ }^{2}$ Department of Geriatric Medicine and Gerontology, Hainan General Hospital, Hainan, China.

${ }^{3}$ Emergency and Critical Care Center, Beijing Anzhen Hospital, Capital Medical University, Beijing, China.

*These authors contributed equally to this work.

(C) Shan Tong et al. 2019; Published by Mary Ann Liebert, Inc. This Open Access article is distributed under the terms of the Creative Commons Attribution Noncommercial License (http://creativecommons.org/licenses/by-nc/4.0/) which permits any noncommercial use, distribution, and reproduction in any medium, provided the original author(s) and the source are cited.
} 
protein beta-catenin ( $\beta$-catenin), co-receptor low-density lipoprotein receptor-related protein 5/6 (LRP5/6), disheveled (DVL), adenomatous polyposis coli (APC), glycogen synthase kinase (GSK)-3 $\beta$, axin, and transcription factors of the $\mathrm{T}$ cell factor/lymphocyte enhancer factor (TCF/LEF). The Wnt protein family consists of a group of proteins that contain cysteine-rich glycosylated ligands and 350-400 amino acid residues, and these proteins are highly conserved signaling molecules exhibiting high degree of homology. Fz consists of $1 \mathrm{~N}$-terminal cysteine-enriched regions [cysteinerich domain (CRD)] as well as 7 transmembrane regions and cytoplasmic regions (cytoplasmic domain) (Kawano and Kypta 2003). The Wnt ligands activate at least 3 different signaling pathways: the canonical Wnt// $\beta$-catenin, the noncanonical Wnt/Jun N-terminal kinase (JNK), and the noncanonical $\mathrm{Wnt} / \mathrm{Ca}^{2+}$ pathways (Pawar and Rao 2018). In the canonical Wnt/ $\beta$-catenin pathway, certain Wnts interact with specific Fz in complex with LRP5/6 to activate the pivotal protein Dvl. Dvl inhibits phosphorylation of cytoplasmic $\beta$-catenin by forming a complex consisting of GSK, APC, and axin. In its nonphosphorylated state, $\beta$-catenin is no longer targeted for degradation. Accumulation of $\beta$-catenin (transferring from the cytoplasm to the nucleus) leads to transcription of the downstream target genes [such as PPAR- $\gamma, \mathrm{C} / \mathrm{EBP} \alpha$, vascular endothelial growth factor (VEGF), TCF-4, and BMP2] via the TCF/LEF promotor. In the noncanonical $\mathrm{Wnt} / \mathrm{JNK}$ pathway, Wnt binds to Frizzled receptors on the surface of cells and subsequently delivers signals into the cells to activate the downstream GTPase Rho and JNK, thus participating in the cytoskeleton activities and regulation of downstream genes via $\mathrm{AP}-1$ promotor (such as TNF- $\alpha$, monocyte chemotactic protein-1, and IL-6). The third pathway is noncanonical $\mathrm{Wnt} / \mathrm{Ca}^{2+}$ pathway. Wnt signals through $\mathrm{Fz}$, in the absence of LRP5/6, to activate G-protein and phospholipase C, which leads to enhanced intracellular $\mathrm{Ca}^{2+}$ levels and activation of protein kinase $\mathrm{C}$ (PKC). Activation of PKC, in turn, leads to the transcription of the downstream target genes (such as IL-6, IL-4, and fibronectin) via the nuclear factor of activated $\mathrm{T}$ cells promotor. In turn, $\mathrm{Ca}^{2+}$-dependent calmodulin kinase is activated and represses the activation of $\beta$-catenin (Fig. 1I).

Sfrps are antagonists that directly bind to Wnt proteins. They have a molecular weight ranging between 30 and $40 \mathrm{kD}$. The CRDs of Sfrps, which lie in the N-terminal half of the protein, are the most important structural characteristic features and consist of 10 conserved cysteine residues and several other conserved segments (Agostino and others 2017). The Cterminal half of Sfrps contains a netrin, which is composed of 6 cysteine residues and several conserved segments of hydrophobic residues. Sfrps represent 5 types of proteins in mammals. Sfrps were initially given several names, reflecting their simultaneous discovery by different approaches, but a unifying nomenclature now exists for 5 of these (Sfrp1-Sfrp5). Sfrps, a modulator protein of Wnt proteins, contains a CRD, which is homologous to that of Wnt receptor, Fz proteins. Thus, Sfrps may block Wnt signaling either by binding to $\mathrm{Fz}$ proteins or by forming nonfunctional complexes with $\mathrm{Fz}$ (Schulte and others 2015). However, Bovolenta and others (2008) confirmed that Sfrps have more than a simple inhibitory effect on the Wnt pathway via other putative mechanisms.

\section{Sfrp5 distribution and biology}

The Sfrp5 gene consists of 317 amino acid residues and 3 coding exons and maps to chromosome 10q24.1 in humans. Sfrp5 was initially found to be highly expressed in retinal pigment epithelium cells and moderately expressed in the pancreas (Chang and others 1999; Satoh and others 2008). In 2010, Ouchi and others (2010) found that Sfrp5 was also highly expressed in white adipose tissue. At present, Sfrp5 is considered to be primarily secreted by adipocytes, especially by visceral adipocytes. However, some scholars have speculated that Sfrp5 may be secreted by the vascular matrix in adipose tissue. The biological function of Sfrp5 primarily includes the regulation of cell polarity and organ formation during embryonic development (Satoh and others 2008; Stuckenholz and others 2013; Fujii and others 2017).

FIG. 1. A signaling map and mechanistic map of the Sfrp5/Wnt signaling pathway in ASCVD. (A) The signaling map of the Wnt signaling pathway. Wnt ligands signal through 3 major pathways. In the canonical Wnt/ $\beta$-catenin (pathway A), certain Wnts interact with specific Fzs in complex with LRP5/6 to activate the pivotal protein Dvl. Dvl inhibits phosphorylation of cytoplasmic $\beta$-catenin by a complex including glycogen synthase kinase-3 $\beta$, adenomatous polyposis coli protein, and axin. In its nonphosphorylated state, $\beta$-catenin is no longer targeted for degradation. The accumulation of $\beta$ catenin (transferring from the cytoplasm to the nucleus) commits to the transcription of the target genes in the downstream (such as PPAR- $\gamma, \mathrm{C} / \mathrm{EBP} \alpha$, VEGF, T cell factor-4, and BMP2) via the T cell factor/lymphoid enhancer factor promotor. In the noncanonical Wnt/Ca2+ (pathway B), Wnt signals through Fz, again in the absence of LRP5/6, activate G-protein and phospholipase $\mathrm{C}$ and lead to raised intracellular Ca2+levels and activation of PKC. The activation of PKC commits to the transcription of the target genes in the downstream (such as IL-6, IL-4, and fibronectin) via the nuclear factor of activated T cells promotor. In turn, $\mathrm{Ca}^{2+}$-dependent calmodulin kinase is activated and represses the activation of $\beta$-catenin. In the noncanonical Wnt/JNK (pathway C): Wnt binds to Frizzled receptors on the surface of cells and subsequently delivers signals into the cells to activate the downstream GTPase Rho and JNK, thus participating in the cytoskeleton and regulation of downstream genes via AP-1 promotor (such as tumor necrosis factor- $\alpha$, monocyte chemotactic protein-1, and IL-6). (B) The mechanistic of physiological and pathological effect of the Wnt signaling pathway in ASCVD. The activation of Wnt signaling pathway could result in neovascularization, vulnerable plaque, myocardial healing, vascular calcification, obesity, dyslipidemia, insulin resistance, inflammation, myocardial remodeling, and myocardial fibrosis and then lead to AS and CVD. Sfrp5 is capable of preventing the binding of Wnt and Frizzled proteins through its combination with Wnt protein, thus blockading the Wnt signaling, inhibiting the transcription of the target genes in the downstream and cytoskeleton. In addition, Sfrp5 has the ability to repress the JNK signaling via its combination with Wnt5a; as a result, it plays a protective effect against AS and ASCVD. VEGF, vascular endothelial growth factor; PKC, protein kinase C; ASCVD, atherosclerotic cardiovascular disease; IL, interleukin; Sfrp, secreted frizzled-related protein; LRP5/6, lipoprotein receptorrelated protein 5/6; Dvl, Disheveled; JNK, Jun N-terminal kinase; AS, atherosclerosis. Color images are available online. 
A Canonical signaling pathway A.Wnt / $\beta$-catenin

B. Wnt $/ \mathrm{Ca}^{2+}$

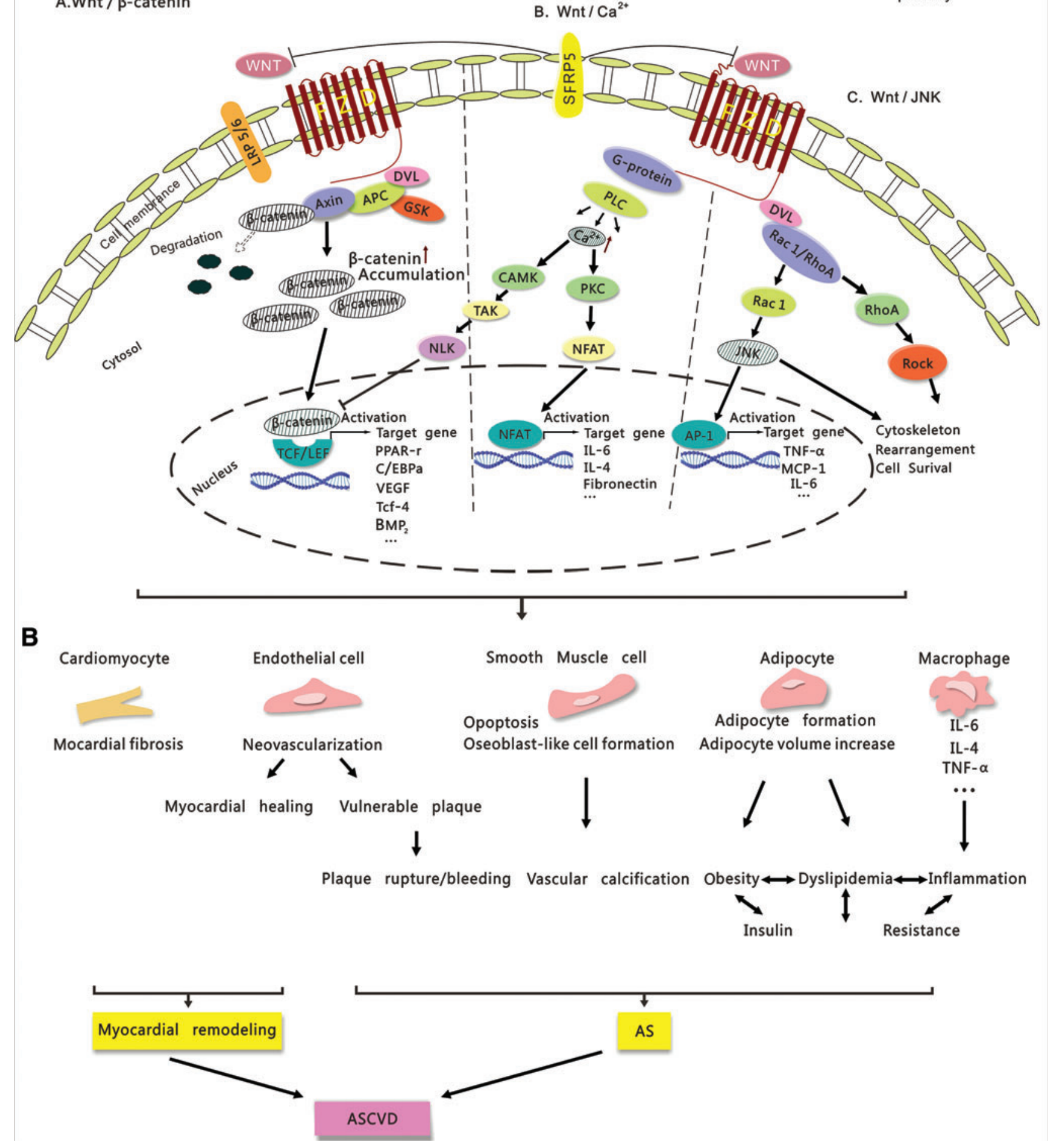

Hypermethylation of Sfrp5 gene contributes to the formation and development of tumors (Maman and others 2011). Sfrp5 has been implicated in the modification of metabolic homeostasis, leading to insulin resistance and obesity-linked metabolic disorders (Ouchi and others 2010; Ouchi 2011) and in the suppressions of inflammatory reactions (Kwon and others 2014; Schulte and others 2015). The effect of Sfrp5 in ASCVD is relatively unexplored; however, several recent studies have suggested that this may be an interesting area to investigate.

\section{Role of Sfrp5/Wnt in the Physiological and Pathological Development of ASCVD}

As is evident, human and murine molecular genetics converge to indicate the important role for Wnt signaling in 
terms of cardiometabolic health (Cheng and others 2015b; Srivastava and others 2015). Wnt signaling plays a major role in the physiological and pathological development of ASCVD, in terms of both metabolic alterations (insulin sensitivity) and cardiovascular remodeling and structural changes (fibrosis, sclerosis, atheroma formation, smooth muscle cell proliferation, and hypertrophy) (Hermans and Blankesteijn 2015). Sfrp5, as faux receptor of Wnt ligands, can restrict Wnt signaling and inactivate specific Wnt actions (Suzuki and others 2004; He and others 2006; Wang and others 2017) (Fig. 1II).

\section{Sfrp5/Wnt and obesity}

Obesity, as an emerging cardiovascular risk factor, is strongly associated with metabolic and cardiovascular disorders. Adipose tissue has been identified as a complex endocrine organ that exerts a wide array of regulatory functions at cellular, tissue, and systemic levels, all of which can have profound effects on the cardiovascular system. Epicardial adipose tissue (EAT), directly surrounding the heart, is a unique and multifaceted fat depot with local and systemic effects (Patel and others 2017). Obesity leads to increased expression of proinflammatory adipokines (such as leptin, adiponectin, and resistin) and inhibited expression of anti-inflammatory adipokines (such as omentin and Sfrp5) in EAT, resulting in the development of a chronic, low-grade inflammatory state (Vural and others 2008; Balta and others 2016). These factors may alter cardiac cell metabolism and functions of endothelial cells, arterial smooth muscle cells, and inflammatory cells, leading to the development of ASCVD (Lastra and Manrique 2015; Patel and others 2017).

Previous studies on the regulation of Sfrp5 in obesity in in vitro show conflicting data. Several groups have reported that Sfrp5 is strongly induced with genetic- and/or dietinduced obesity in mice (Koza and others 2006; Lagathu and others 2009; Okada and others 2009; Mori and others 2012). However, Ouchi and others (2010) reported very different findings from the previous literature. They found that Sfrp5 gene expression downregulated when the volume of adipocytes increased to the platform stage, indicating that there may be a feedback regulation of Sfrp5 expression and adipocyte volume. The authors pointed out the transient role of Sfrp5, suggesting that Sfrp5 expression is increased in adipose tissue but is reduced in response to serious obesityrelated metabolic dysfunctions. This proved that the upregulation of Sfrp5 in obese mice alleviates metabolic disorders and suppresses inflammation. In our opinion, these discrepancies may be accounted for by the different animal models, in terms of age of the animals at the time of detection, and different measurements. Previous studies have shown that the Wnt signaling pathway can inhibit the formation of adipose tissue by suppressing PPAR- $\gamma$ function and C/EBP $\beta$ (CCATT enhancer-binding protein beta) (Takada and others 2007; Tang and Lane 2012). Inhibiting the Wnt signaling pathway in myocytes can induce myoblasts to transform into adipocytes (Guo and others 2012). Through both canonical Wnt pathways and noncanonical Wnt pathways, GSK activates STAT5 (signal transducer and activator of transcription 5) phosphorylation to bind to the promoter of Sfrp genes, and PPAR- $\gamma$ gene, to stimulate Sfrp5 gene expression, which could ultimately lead to a modulated adipo- genic process (Wang and others 2018). Mori and others (2012) found that adipose tissue from Sfrp5-deficient mice exhibited similar number of adipocytes, indicating that Sfrp5 did not affect the proliferation of adipocytes. Nevertheless, the number of large fat cells in these mice was less than that in wild-type mice, suggesting that Sfrp5 affects adipocyte hypertrophy. Through further studies on molecular mechanisms, the authors supported a model of adipogenesis in which Sfrp5 inhibits Wnt signaling to suppress oxidative metabolism and stimulates adipocyte growth during obesity.

In contrast with in vitro studies, Sfrp5 plasma levels were also shown to be lower than those in lean participants and were negatively correlated with the markers of obesity, such as body mass index (BMI) and waist circumference (Schulte and others 2012; Hu and others 2013b; Tan and others 2014). Reduced circulating Sfrp5 levels may play a role in the etiopathogenesis of obesity. Furthermore, decreases in weight through life intervention significantly correlated with the upregulation of Sfrp5 (Schulte and others 2012; Tan and others 2014). Schulte and others (2012) showed that Sfrp5 expression is not significantly different between obese and lean individuals, unlike Wnt5a, which is significantly upregulated in obese subjects. To date, the first study to investigate the polymorphisms in the Sfrp5 gene was performed in a large population cohort of obese and nonobese individuals (Van Camp and others 2016). The results showed that Sfrp5 single nucleotide polymorphisms (c.-3G $>$ A) are associated with BMI. The Sfrp5 rs7072751 in male obese patients is associated with obesity, which can explain the $1.8 \%$ variance of abdominal obesity in general population. The roles of Sfrp5 in weight loss need to be further elucidated.

\section{Sfrp5/Wnt and insulin resistance}

Type 2 diabetes (T2D) is considered to be an equivalent risk factor of CVD. Abnormalities in insulin resistance and glucose metabolism play a key role in the development of AS and CVD. The Wnt pathway and related signaling factors, including Sfrp5, are important in the development of diabetes and insulin resistance. It is generally considered that the Wnt signaling pathway participates in the proliferation of $\beta$ cells; however, $\beta$-catenin may also play an important role in this process (Rulifson and others 2007; Rebuffat and others 2013). Several in vitro experiments have shown that the level of Sfrp5 was significantly reduced when insulin levels were high (Lv and others 2012; Guan and others 2016); however, Sfrp5 expression upregulated upon treatment with rosiglitazone and metformin ( $\mathrm{Lv}$ and others 2012). Sfrp5-/- mice exhibited glucose metabolic dysfunction when fed a high-calorie diet compared with wild-type mice (Ouchi and others 2010). In contrast, in in vitro conditions (Mori and others 2012), the silencing of Sfrp5 did not influence signaling events implicated in insulin resistance (including the phosphorylation of Ser307IRS1 or JNK) and glucose uptake in the primary or immortalized adipocytes. These data provided no support for the hypothesis that Wnt5a or Sfrp5 regulates the insulin sensitivity of adipocytes. The disparity in results may be due to the use of different models and different detection times. Therefore, although the pleiotropic activity and insulinsensitizing effect of Sfrp5 have been demonstrated, its specific mechanism remains unclear. 
Circulating Sfrp5 levels were examined with respect to abnormal glucose metabolism in clinical cross-sectional studies in humans. Three studies on Chinese subjects reported that circulating Sfrp5 levels were lower in individuals with impaired glucose tolerance and T2D than in those with normal glucose tolerance (Hu and others 2013b; Cheng and others 2015a). Moreover, circulating Sfrp5 level is negatively correlated with BMI, waist-to-hip ratio, and homeostatic model assessment for insulin resistance (Hu and others 2013b; Cheng and others 2015a). In addition, decreased serum Sfrp5 is more closely related to the function of $\beta$ cells than insulin sensitivity (CarstensenKirberg and others 2016). Furthermore, after the application of liraglutide, the level of Sfrp5 increased accordingly with improved blood glucose control and insulin resistance (Hu and others 2013a). Recently, the German KORA F4 study investigated a cross-sectional association between serum Sfrp5 level and cardiometabolic risk factors, and prediabetes/T2D, in 1,096 participants, 666 of whom had prediabetes or T2D (Carstensen-Kirberg and others 2017). The results showed that serum Sfrp5 levels were lower in participants with prediabetes or T2D than in those with normal glucose tolerance. In the fully adjusted model, higher Sfrp5 level was associated with lower incidence of prediabetes/T2D [odds ratio (OR) $0.72 ; 95 \%$ confidence interval (CI) 0.58-0.89], and this association was independent of BMI. Higher serum Sfrp5 level was inversely correlated with multiple risk factors for T2D and CVD, suggesting that it may be a novel biomarker of CVD. However, the aforementioned data are in contrast to 2 other studies ( $\mathrm{Lu}$ and others 2013; Canivell and others 2015), both of which showed that serum Sfrp5 level was upregulated in T2D and that serum Wnt5a level was reduced (Lu and others 2013). In the second study (Canivell and others 2015), after adjusting for potential confounders [age, gender, BMI, triglycerides, high-density lipoprotein cholesterol (HDL-C), and blood pressure], T2D was still associated with higher levels of Sfrp5 compared with prediabetes as revealed by multinomial logistic regression analysis (OR 3.50; 95\% CI 1.40-8.79). The association was more subtle when comparing with the normal glucose tolerance state (OR 2.18; 95\% CI 0.91-5.21). Such inconsistencies were mainly caused by different subjects (patients and controls), detection methods, and sample size. Above all, the exact roles of Sfrp5 in insulin resistance need to be further investigated.

\section{Sfrp5/Wnt and lipid metabolism}

Lipid metabolism disorder is key part of AS and is an important risk factor for ASCVD. Increasing evidence suggests that Sfrp5 may be a protective cytokine associated with lipid metabolism. In an animal study, Sfrp5-deficient mice were metabolically normal when maintained on a regular diet but displayed increased fatty liver disease when fed a high-calorie diet for 12 weeks. After Sfrp5 treatment, the accumulation of lipids in the liver decreased, and the degree of fatty liver disease was reversed (Ouchi and others 2010). Serum Sfrp5 level was significantly decreased in rats that were fed a high-fat diet, whereas dephosphorylated beta protein levels increased compared with the total levels of beta protein in islet cells, suggesting that Sfrp5 silencing promotes $\beta$ cell proliferation by activating the canonical Wnt pathway (Rebuffat and others 2013). Meanwhile, population-based studies have suggested that Sfrp5 functions to protect lipid metabolism. Serum Sfrp5 level was negatively correlated with triglycerides ( $\mathrm{Hu}$ and others 2013a; Xu and others 2017), free fatty acids ( $\mathrm{Hu}$ and others 2013a), low-density lipoprotein cholesterol (Almario and Karakas 2015), and apolipoprotein B (Almario and Karakas 2015) and was positively correlated with HDL-C (Hu and others 2013a; Xu and others 2017). However, no significant correlation was found between the plasma Sfrp5 level and lipid metabolism in another study (Carstensen and others 2013). Nevertheless, the increasing number of recent studies suggests that Sfrp5 plays a role in improving lipid metabolism, but its mechanism of action has yet to be clarified.

\section{Sfrp5/Wnt and inflammation}

Accumulating evidence indicates that the obese state is characterized by chronic low-grade inflammation. In the human body, Wnt5a is expressed by macrophages in adipose tissue and may be an important proinflammatory factor associated with low inflammation (Festa and others 2000; Zuriaga and others 2017). As an inhibitor of Wnt5a, Sfrp5 inhibits the activation of downstream molecules of the Wnt pathway and reduces the secretion of inflammatory factors (Ouchi and others 2010). On one hand, decreased Sfrp5 level during obesity is associated with inflammation, indicated by increased TNF- $\alpha$ and IL- 6 levels and activation of toll-like receptor-4 and nuclear factor light-chain kappa B signaling pathways. Furthermore, the activation of these adipocytokines promotes weight loss and reduces inflammation (Festa and others 2000). On the other hand, obese patients exhibited increased Wnt5a mRNA levels in visceral adipose tissue and downregulated Sfrp5 gene expression. The expression of Wnt5a mRNA was significantly enhanced by lipopolysaccharide and TNF- $\alpha$ treatment, and exogenous Wnt5a treatment induced the expression of IL-6, IL-1B, MMP2, MMP9, and SSP1 mRNA in human adipocytes (Catalan and others 2014). Taken together, the activation of noncanonical Wnt signaling through upregulation of Wnt5a and downregulation of Sfrp5 may promote a proinflammatory state in visceral adipose tissue, contributing to the development of ASCVD.

The Sfrp5/Wnt5a-mediated noncanonical pathways are associated with the pathogenesis of many inflammationrelated diseases. First, Wnt5a levels were found to be significantly increased in septic patients compared with healthy controls and exhibited a significant positive correlation with the leukocyte count. Interestingly, in patients recovering from sepsis, Wnt5a levels significantly declined. In contrast, as sepsis progressed, Wnt5a levels increased in a timedependent manner (Schulte and others 2015). Second, Kwon and others (2014) revealed that Sfrp5 suppressed the inflammatory response by preventing JNK activation in rheumatoid arthritis, which proved the anti-inflammatory effect of Sfrp5. Third, several clinical studies have reported the association between decreased Sfrp5 levels and inflammation in T2D patients (Hu and others 2013b; Almario and Karakas 2015). Fourth, human periodontitis was associated with high expression level of Wnt5a and low expression level of Sfrp5, although this expression profile was reversed after treatment with Sfrp5 in mouse model (Maekawa and others 2017). The role of the Sfrp5/Wnt5a regulatory system in CVD is, thus far, relatively unexplored; however, several 
recent studies have suggested that this topic may be an interesting area to investigate (Kitagawa and others 2017). In contrast, Carstensen and others (2013) obtained contradictory results, that is, there is no significant correlation between serum Sfrp5 levels and inflammation markers. Another study demonstrated that the cellular actions of Sfrp5 seem to depend on the type of tissue as well as its inflammatory and metabolic states. Sfrp5 inhibited IL-6 release in TNF-treated human adipocytes but did not act on skeletal muscle cells (Carstensen and others 2014). Therefore, further studies on the relationship between Wnt pathway, its inhibitor Sfrp5, and inflammation are needed.

\section{Sfrp5/Wnt and fibrosis}

The Wnt pathway regulates the process of fibrosis in different organs. Sfrps, as an inhibitor of Wnt, plays an important role in inhibiting fibrosis (He and others 2010; Matsushima and others 2010). Wnt5a mediates the differentiation of adipocytes into fibroblasts in the pancreas that may create a microenvironment for carcinoma (Zoico and others 2016). Moreover, Sfrp5 can alleviate liver fibrosis in mice by inhibiting Wnt5a/Fz2 (Chatani and others 2015). In addition, indoxyl sulfate-induced renal fibrosis occurred due to Sfrp5 hypermethylation in chronic kidney disease mice. Sfrp5 upregulation attenuated renal fibrosis by inhibiting Wnt signaling (Yu and others 2017). Finally, several studies have demonstrated that the transfection of miR-125b mimics into cardiac fibroblasts (CFs) resulted in significantly increased expression of the myofibroblast markers, alphasmooth muscle actin ( $\alpha$-SMA), and vinculin, whereas the transfection of Sfrp5 resulted in the opposite effect. Sfrp5 inhibited the expression of $\alpha$-SMA and collagen I and III in $\mathrm{CFs}$. Further analysis revealed that miR-125b promotes the proliferation and migration of CFs and inhibits their apo- ptosis, whereas Sfrp5 exhibits the opposite effect (Bie and others 2016).

\section{Role of Sfrp5/Wnt in ASCVD}

Wnt signals act not only as regulators of the dysmetabolic milieu driving CVD but also as mediators of CVD in response to the dysmetabolic milieu (Matsushima and others 2010; Gay and Towler 2017). Sfrps, the family of Wnt antagonists, exhibit different functions in the progression of CVD (Barandon and others 2003; Ezan and others 2004; Mirotsou and others 2007; Kobayashi and others 2009; Matsushima and others 2010; Mastri and others 2014; Miyoshi and others 2014; Lin and others 2016; Nakamura and others 2016; Ji and others 2017b) (Table 1). Similarly, Sfrp5 inhibits the binding of Wnt and Frizzled proteins by binding with Wnt protein and thus suppressing the Wnt signaling, inhibiting the transcription of the downstream target genes and cytoskeleton. In addition, Sfrp5 suppresses JNK signaling by binding with Wnt5a and thus exhibits a protective effect against AS and ASCVD (Fig. 1I).

\section{Sfrp5/Wnt in AS}

Sfrp5 participates in the pathogenesis of AS, such as inflammatory reactions, cell proliferation, and macrophage chemotaxis. Sfrp5 levels are significantly lower in patients with obesity, insulin resistance, and diabetes, which lead to the development of metabolic syndrome, AS, and autoimmune disorders (Jaikanth and others 2017). Christman and others (2008) first confirmed the hypothesis that Wnt5a plays a role in AS pathogenesis in vitro. Sfrp5, an antagonist of Wnt5a, suppressed the activation of JNK in adipose tissue and macrophages and inhibited the release of

Table 1. Summary of Some Findings Related to Sfrps in Cardiovascular Disease

\begin{tabular}{|c|c|c|c|}
\hline Sfrps & Subject & Finding & Study \\
\hline \multirow[t]{2}{*}{ Sfrp1 } & Mice & $\begin{array}{l}\text { Overexpression of Sfrp1 reduces myocardial infarction size and improves cardiac } \\
\text { function }\end{array}$ & $\begin{array}{l}\text { Barandon and others } \\
\text { (2003) }\end{array}$ \\
\hline & Mice & Reduces endothelial and vascular smooth muscle cell proliferation & $\begin{array}{l}\text { Ezan and others } \\
\text { (2004) }\end{array}$ \\
\hline \multirow[t]{4}{*}{ Sfrp2 } & Mice & Promoting myocardial stem cell survival and repair with ischemia & $\begin{array}{l}\text { Mirotsou and others } \\
\text { (2007) }\end{array}$ \\
\hline & Mice & Antagonism in controlling fibrosis in the infarcted heart & $\begin{array}{l}\text { Kobayashi and } \\
\text { others }(2009)\end{array}$ \\
\hline & Mice & $\begin{array}{l}\text { Sfrp } 2 \text { blockade increased myocardial levels of vascular endothelial growth factor and } \\
\text { hepatocyte growth factor along with increased angiogenesis }\end{array}$ & $\begin{array}{l}\text { Mastri and others } \\
\quad(2014)\end{array}$ \\
\hline & Mice & $\begin{array}{l}\text { Srp } 2 \text { may regulate the growth and extracellular matrix remodeling of adult mouse } \\
\text { cardiac fibroblasts after myocardial infarction }\end{array}$ & $\begin{array}{l}\text { Lin and others } \\
(2016)\end{array}$ \\
\hline \multirow[t]{2}{*}{ Sfrp4 } & Mice & $\begin{array}{l}\text { Administration of Sfrp4 interferes could mediate the formation of acellular scar and } \\
\text { consequently contributes to the prevention of aggravation of cardiac function }\end{array}$ & $\begin{array}{l}\text { Matsushima and } \\
\text { others (2010) }\end{array}$ \\
\hline & Human & $\begin{array}{l}\text { EAT-derived and circulating Sfrp4 expression levels were increased in patients with } \\
\text { CAD. EAT Sfrp4 mRNA levels and plasma Sfrp4 concentrations were } \\
\text { independently associated with the presence of CAD }\end{array}$ & Ji and others $(2017 b)$ \\
\hline \multirow[t]{3}{*}{ Sfrp5 } & Mice & $\begin{array}{l}\text { Inhibits myocardial inflammation and injury in a preclinical ischemia/reperfusion } \\
\text { mode }\end{array}$ & $\begin{array}{l}\text { Nakamura and others } \\
\quad(2016)\end{array}$ \\
\hline & Human & $\begin{array}{l}\text { Serum SfrpP5 levels were significantly associated with CAD in humans, suggesting } \\
\text { that low Sfrp5 levels may contribute to CAD }\end{array}$ & $\begin{array}{l}\text { Miyoshi and others } \\
\text { (2014) }\end{array}$ \\
\hline & Human & $\begin{array}{l}\text { A high serum concentration of Sfrp5 was associated with the occurrence of future } \\
\text { cardiovascular events, especially in the elderly patients }\end{array}$ & Ji and others $(2017 a)$ \\
\hline
\end{tabular}


proinflammatory cytokines mediated by Wnt5a. Peripheral artery disease is associated with the elevation in levels of an anti-angiogenic, VEGF-A splice isoform (VEGFA165b), and a corresponding reduction in levels of the proangiogenic, VEGF-A165a splice isoform, which is the primary indicator of AS. In mice, VEGF-A165b expression was upregulated by the genetic ablation of Sfrp5 (Kikuchi and others 2014). Malgor and others (2014) assessed Wnt5a expression in different sections of atherosclerotic plaque tissues isolated from human subjects undergoing elective carotid endarterectomy. The data analysis revealed that Wnt5a transcripts and proteins were elevated in more advanced arterial lesions relative to less advanced arterial lesions and that the average amount of Wnt5a protein present in atherosclerotic patient serum was significantly higher than those in the healthy controls. Recently, a casecontrol study showed that the incidence and severity of coronary AS are also associated with Sfrp5 levels (Miyoshi and others 2014). A Korean study showed that the serum level of Sfrp5 is associated with AS in humans with T2D mellitus. Furthermore, in the in vitro study, they reported that Sfrp5 ameliorated Wnt5a-induced endothelial dysfunction (Cho and others 2018).

Plaque rupture or bleeding is an important cause of severe events of AS or other complications, and the stability of plaque is closely related to the density of the neovascularization in the lesion. Meanwhile, the number of new vessels in the plaques increases significantly during moderate and severe inflammation, and the degree of new vessel formation was the highest in ruptured plaques, indicating that neovascularization is closely related to plaque inflammation. During the early stage of angiogenesis, consisting of endothelial differentiation and other pathophysiological activities, Wnt protein is secreted as a signaling protein that can control various cell life activities, such as cell differentiation and proliferation. Functional studies indicate that the $\mathrm{Wnt} / \beta$-catenin pathway, which can be detected in the vascular system, is needed for angiogenesis, suggesting that suppressing its expression would inhibit angiogenesis. The $\mathrm{Wnt} / \beta$-catenin pathway can promote retinal vascularization and is accompanied by the enhancement of $\beta$-catenin expression in the nucleus. The target gene of Wnt can encode angiogenic factor VEGF and regulate endothelial cells and angiogenesis, which further validates the role of this pathway in the vascular system. Considering that Sfrp5 is an inhibitor of the Wnt pathway, it is possible that $\mathrm{Sfrp} 5$ can reduce the occurrence of neovascularization in plaques and inhibit the rupture and bleeding of plaques.

Vascular calcification is another important characteristic of AS and is positively correlated with the size of the plaque. During vascular calcification, vascular smooth muscle cells (VSMCs) undergo apoptosis or are transformed into osteoblast-like cells, and then, calcium and phosphorus become concentrated in the extracellular matrix and promote calcification. Osteoblast differentiation can be regulated by the $\mathrm{Wnt} / \beta$-catenin signaling pathway, where $\beta$-catenin and BMP2 play an important role. An in vitro study in human VSMCs (Gherghe and others 2011), cultivated in high phosphate (HP) medium, showed that the translocation of $\beta$-catenin into the nucleus was enhanced and that the expression of osteogenic factors, such as BMP2, Msx2, and osteocalcin, was upregulated
(Gherghe and others 2011). These changes can be inhibited by DKK-1, a specific natural antagonist of the Wnt/ $\beta$-catenin signaling pathway. Furthermore, the addition of Sfrp5 significantly inhibited HP-induced calcification of VSMCs, which was recovered by anti-Sfrp5 treatment. In addition, Sfrp5 abrogated HP-induced activation via the Wnt/ $\beta$-catenin pathway (Deng and others 2016). These results suggested that Sfrp5 inhibits the calcification of VSMCs as well as the Wnt/ $\beta$-catenin pathway.

Therefore, Sfrp5 might have protective effects against AS, plaque stability, and vascular calcification. However, future studies are needed to further elucidate the significance of these discoveries.

\section{Sfrp5/Wnt in coronary artery disease}

To date, there are 2 clinical correlation studies related to circulating Sfrp5 in coronary artery disease (CAD), which reached opposite conclusions. A case-control study from Japan (Miyoshi and others 2014) showed that serum Sfrp5 levels in CAD patients, compared with those in non-CAD individuals, were lower and closely related to BMI, insulin resistance, adiponectin level, and $\mathrm{CAD}$ sensitivity. In the multivariate logistic regression analysis, the serum Sfrp5 level was an independent decisive factor for the presence of CAD (OR 0.36; 95\% CI 0.14-0.94). It was confirmed that serum Sfrp5 level is closely related to CAD, and low serum Sfrp5 levels may lead to CAD incidence. In addition, the levels of Sfrp5 were even lower in patients with multiple lesions than those with single-branch lesions, and the Gensini score was negatively correlated with Sfrp5 levels, suggesting that Sfrp5 was associated with the severity of CAD. However, the aforementioned results were opposite to those obtained after a 4-year follow-up prospective study on 168 Chinese individuals ( $\mathrm{Ji}$ and others 2017a), which investigated whether Sfrp5 is associated with future cardiovascular events in both $\mathrm{CAG}(+)$ and $\mathrm{CAG}(-)$ subgroups in terms of a composite primary endpoint of combined occurrence of major adverse cardiovascular events (MACE). The result showed that a high serum concentration of Sfrp5 is associated with the occurrence of future cardiovascular events, especially in the elderly patients. In the multivariate Cox regression analysis, high Sfrp5 significantly predicted patients' MACE with an hazard ratio (HR) of $2.174(95 \%$ CI: 1.169-4.041) at 2-year follow-up and an HR of 1.974 (95\% CI: 1.138-3.426) at 4-year follow-up. The incidence of MACE was significantly increased in the high Sfrp5 expression group for $\mathrm{CAG}(-)$ patients, as well as for those older than 65 years. In our opinion, the opposing result may be mainly due to the different types of study: case-control study versus prospective study. Recently, in a Northern Shanghai study (involving 1,745 community-dwelling subjects aged older than 65 years from northern China) reported that plasma Sfrp5 level was inversely correlated with conventional cardiovascular risk factors, and low plasma Sfrp5 level was also significantly associated with asymptomatic hypertensive target organ damages in the elderly Chinese population (Teliewubai and others 2018). Thus, Sfrp5 may potentially become a future therapeutic target as well as a potential biomarker of CVD.

The mechanism of Sfrp5 in CAD is still unclear. Animal studies have shown that the Wnt pathway can inhibit the incidence of coronary heart disease (Miyoshi and others 
2014). However, the cause-or-effect relationship between Sfrp5 and CAD has not been established and should be explored in future studies.

\section{Conclusions}

To summarize, here we reviewed the recent progress made in the relationship between Sfrp5 and ASCVD. Although both in vitro and in vivo studies proved that Sfrp5 is involved in the pathological process of obesity, insulin resistance, dyslipidemia, inflammation, and fibrosis and is associated with obesity, T2D, and ASCVD, the results are still conflicting. These discrepancies may arise due to diverse reasons: on one hand, Sfrp5 level may have a cutoff effect on glucose and lipid metabolism; on the other hand, such discrepancy may be related to the different study design, inclusion criteria of the study subjects, measurements conducted, and the sample size of study. In addition, systemic and local effects of Sfrp5 are different because the in vitro study concentrated on the impact of Sfrp5 in cell and did not consider its interaction with other cells as well as potential locally occurring binding partners. As a secreted protein, circulatory Sfrp5 has been shown to be responsible for ASCVD and conventional cardiovascular risk factors. Therefore, we propose that Sfrp5 could be exploited as a novel biomarker for early detection of ASCVD. In vitro experiments have demonstrated that treatment with recombinant Sfrp5 reduces the ischemic myocardial injuries, which indicated that it may be a promising novel therapeutic target. The potential therapeutic role of Sfrp5 in ASCVD and the identification of Sfrp5 receptors will be challenging topics for future investigations.

\section{Acknowledgments}

This work was supported by the Beijing Municipal Administration of Hospitals Clinical Medicine Development of Special Funding Support (code: ZYLX201303, XMLX201601), the grant from the National Key Research and Development Program of China (2017YFC0908800) and the Beijing Municipal Administration of Hospitals' Ascent Plan (code: DFL20150601) and Mission Plan (code: SML20180601).

\section{Author Disclosure Statement}

No competing financial interests exist.

\section{References}

Agostino M, Pohl SO, Dharmarajan A. 2017. Structurebased prediction of Wnt binding affinities for Frizzledtype cysteine-rich domains. J Biol Chem 292(27): 11218-11229.

Almario RU, Karakas SE. 2015. Roles of circulating WNTsignaling proteins and WNT-inhibitors in human adiposity, insulin resistance, insulin secretion, and inflammation. Horm Metab Res 47(2):152-157.

Balta S, Demir M, Yildirim AO, Demirkol S, Ozturk C, Celik T. 2016. Epicardial fat thickness and cardiovascular involvements. Afr Health Sci 15(4):1354-1355.

Barandon L, Couffinhal T, Ezan J, Dufourcq P, Costet P, Alzieu P, Leroux L, Moreau C, Dare D, Duplaa C. 2003. Reduction of infarct size and prevention of cardiac rupture in transgenic mice overexpressing FrzA. Circulation 108(18):2282-2289.

Bie ZD, Sun LY, Geng CL, Meng QG, Lin XJ, Wang YF, Wang XB, Yang J. 2016. MiR-125b regulates SFRP5 expression to promote growth and activation of cardiac fibroblasts. Cell Biol Int 40(11):1224-1234.

Bovolenta P, Esteve P, Ruiz JM, Cisneros E, Lopez-Rios J. 2008. Beyond Wnt inhibition: new functions of secreted Frizzled-related proteins in development and disease. J Cell Sci 121(Pt 6):737-746.

Canivell S, Rebuffat S, Ruano EG, Kostov B, Siso-Almirall A, Novials A, Ceriello A, Gomis R. 2015. Circulating SFRP5 levels are elevated in drug-naive recently diagnosed type 2 diabetic patients as compared with prediabetic subjects and controls. Diabetes Metab Res Rev 31(2):212219.

Carstensen M, Herder C, Kempf K, Erlund I, Martin S, Koenig W, Sundvall J, Bidel S, Kuha S, Roden M, et al. 2013. Sfrp5 correlates with insulin resistance and oxidative stress. Eur J Clin Invest 43(4):350-357.

Carstensen M, Wiza C, Rohrig K, Fahlbusch P, Roden M, Herder C, Ouwens DM. 2014. Effect of Sfrp5 on cytokine release and insulin action in primary human adipocytes and skeletal muscle cells. PLoS One 9(1):e85906.

Carstensen-Kirberg M, Hatziagelaki E, Tsiavou A, Chounta A, Nowotny P, Pacini G, Dimitriadis G, Roden M, Herder C. 2016. Sfrp5 associates with beta-cell function in humans. Eur J Clin Invest 46(6):535-543.

Carstensen-Kirberg M, Kannenberg JM, Huth C, Meisinger C, Koenig W, Heier M, Peters A, Rathmann W, Roden M, Herder C, et al. 2017. Inverse associations between serum levels of secreted frizzled-related protein-5 (SFRP5) and multiple cardiometabolic risk factors: KORA F4 study. Cardiovasc Diabetol 16(1):109.

Catalan V, Gomez-Ambrosi J, Rodriguez A, Perez-Hernandez AI, Gurbindo J, Ramirez B, Mendez-Gimenez L, Rotellar F, Valenti V, Moncada R, et al. 2014. Activation of noncanonical Wnt signaling through WNT5A in visceral adipose tissue of obese subjects is related to inflammation. J Clin Endocrinol Metab 99(8):E1407-E1417.

Chang JT, Esumi N, Moore K, Li Y, Zhang S, Chew C, Goodman B, Rattner A, Moody S, Stetten G, et al. 1999. Cloning and characterization of a secreted frizzled-related protein that is expressed by the retinal pigment epithelium. Hum Mol Genet 8(4):575-583.

Chatani N, Kamada Y, Kizu T, Ogura S, Furuta K, Egawa M, Hamano M, Ezaki H, Kiso S, Shimono A, et al. 2015. Secreted frizzled-related protein 5 (Sfrp5) decreases hepatic stellate cell activation and liver fibrosis. Liver Int 35(8): 2017-2026.

Cheng L, Zhang D, Chen B. 2015a. Declined plasma sfrp5 concentration in patients with type 2 diabetes and latent autoimmune diabetes in adults. Pak J Med Sci 31(3):602605.

Cheng SL, Ramachandran B, Behrmann A, Shao JS, Mead M, Smith C, Krchma K, Bello AY, Kovacs A, Kapoor K, et al. 2015b. Vascular smooth muscle LRP6 limits arteriosclerotic calcification in diabetic LDLR-/- mice by restraining noncanonical Wnt signals. Circ Res 117(2):142-156.

Cho YK, Kang YM, Lee SE, Lee Y, Seol SM, Lee WJ, Park JY, Jung CH. 2018. Effect of SFRP5 (Secreted Frizzled-Related Protein 5) on the WNT5A (Wingless-Type Family Member 5A)-induced endothelial dysfunction and its relevance with arterial stiffness in human subjects. Arterioscler Thromb Vasc Biol 38(6):1358-1367. 
Christman MN, Goetz DJ, Dickerson E, McCall KD, Lewis CJ, Benencia F, Silver MJ, Kohn LD, Malgor R. 2008. Wnt5a is expressed in murine and human atherosclerotic lesions. Am J Physiol Heart Circ Physiol 294(6):H2864$\mathrm{H} 2870$.

Deng D, Diao Z, Han X, Liu W. 2016. Secreted frizzled-related protein 5 attenuates high phosphate-induced calcification in vascular smooth muscle cells by inhibiting the Wnt/ß-catenin pathway. Calcif Tissue Int 99(1):66-75.

Ezan J, Leroux L, Barandon L, Dufourcq P, Jaspard B, Moreau C, Allieres C, Daret D, Couffinhal T, Duplaa C. 2004. FrzA/ sFRP-1, a secreted antagonist of the Wnt-Frizzled pathway, controls vascular cell proliferation in vitro and in vivo. Cardiovasc Res 63(4):731-738.

Festa A, D’Agostino RJ, Howard G, Mykkanen L, Tracy RP, Haffner SM. 2000. Chronic subclinical inflammation as part of the insulin resistance syndrome: the Insulin Resistance Atherosclerosis Study (IRAS). Circulation 102(1): 42-47.

Fujii M, Sakaguchi A, Kamata R, Nagao M, Kikuchi Y, Evans SM, Yoshizumi M, Shimono A, Saga Y, Kokubo H. 2017. Sfrp5 identifies murine cardiac progenitors for all myocardial structures except for the right ventricle. Nat Commun 8: 14664.

Gay A, Towler DA. 2017. Wnt signaling in cardiovascular disease: opportunities and challenges. Curr Opin Lipidol 28(5):387-396.

Gherghe CM, Duan J, Gong J, Rojas M, Klauber-Demore N, Majesky M, Deb A. 2011. Wnt1 is a proangiogenic molecule, enhances human endothelial progenitor function, and increases blood flow to ischemic limbs in a HGF-dependent manner. FASEB J 25(6):1836-1843.

Guan B, Li W, Li F, Xie Y, Ni Q, Gu Y, Li X, Wang Q, Zhang H, Ning G. 2016. Sfrp5 mediates glucose-induced proliferation in rat pancreatic beta-cells. J Endocrinol 229(2): 73-83.

Guo B, Chatterjee S, Li L, Kim JM, Lee J, Yechoor VK, Minze LJ, Hsueh W, Ma K. 2012. The clock gene, brain and muscle Arnt-like 1, regulates adipogenesis via Wnt signaling pathway. FASEB J 26(8):3453-3463.

He J, Sheng T, Stelter AA, Li C, Zhang X, Sinha M, Luxon BA, Xie J. 2006. Suppressing Wnt signaling by the hedgehog pathway through sFRP-1. J Biol Chem 281(47):3559835602.

He W, Zhang L, Ni A, Zhang Z, Mirotsou M, Mao L, Pratt RE, Dzau VJ. 2010. Exogenously administered secreted frizzled related protein 2 (Sfrp2) reduces fibrosis and improves cardiac function in a rat model of myocardial infarction. Proc Natl Acad Sci U S A 107(49):21110-21115.

Hermans KC, Blankesteijn WM. 2015. Wnt signaling in cardiac disease. Compr Physiol 5(3):1183-1209.

Hu W, Li L, Yang M, Luo X, Ran W, Liu D, Xiong Z, Liu H, Yang G. 2013a. Circulating Sfrp5 is a signature of obesityrelated metabolic disorders and is regulated by glucose and liraglutide in humans. J Clin Endocrinol Metab 98(1):290 298.

Hu Z, Deng H, Qu H. 2013b. Plasma SFRP5 levels are decreased in Chinese subjects with obesity and type 2 diabetes and negatively correlated with parameters of insulin resistance. Diabetes Res Clin Pract 99(3):391-395.

Jaikanth C, Gurumurthy P, Indhumathi T, Cherian KM. 2017. Emergence of SFRP5 as a pleiotropic adipocytokine and its association with Wnt signaling pathways. Minerva Endocrinol 42(3):280-289.
Ji H, Li H, Zhuang J, Su Y, Wen J. 2017a. High serum level of secreted frizzled-related protein 5 (sfrp5) is associated with future cardiovascular events. Cardiovasc Ther 2(1): e115.

Ji Q, Zhang J, Du Y, Zhu E, Wang Z, Que B, Miao H, Shi S, Qin X, Zhao Y, et al. 2017b. Human epicardial adipose tissue-derived and circulating secreted frizzledrelated protein 4 (SFRP4) levels are increased in patients with coronary artery disease. Cardiovasc Diabetol 16(1): 133.

Kawano Y, Kypta R. 2003. Secreted antagonists of the Wnt signalling pathway. J Cell Sci 116(Pt 13):2627-2634.

Kikuchi R, Nakamura K, MacLauchlan S, Ngo DT, Shimizu I, Fuster JJ, Katanasaka Y, Yoshida S, Qiu Y, Yamaguchi TP, et al. 2014. An antiangiogenic isoform of VEGF-A contributes to impaired vascularization in peripheral artery disease. Nat Med 20(12):1464-1471.

Kitagawa T, Yamamoto H, Hattori T, Sentani K, Takahashi S, Senoo A, Kubo Y, Yasui W, Sueda T, Kihara Y. 2017. Tumor necrosis factor-alpha gene expression in epicardial adipose tissue is related to coronary atherosclerosis assessed by computed tomography. J Atheroscler Thromb 25(3):269280.

Kobayashi K, Luo M, Zhang Y, Wilkes DC, Ge G, Grieskamp T, Yamada C, Liu TC, Huang G, Basson CT, et al. 2009. Secreted Frizzled-related protein 2 is a procollagen $\mathrm{C}$ proteinase enhancer with a role in fibrosis associated with myocardial infarction. Nat Cell Biol 11(1):46-55.

Koza RA, Nikonova L, Hogan J, Rim JS, Mendoza T, Faulk C, Skaf J, Kozak LP. 2006. Changes in gene expression foreshadow diet-induced obesity in genetically identical mice. PLoS Genet 2(5):e81.

Kwon YJ, Lee SW, Park YB, Lee SK, Park MC. 2014. Secreted frizzled-related protein 5 suppresses inflammatory response in rheumatoid arthritis fibroblast-like synoviocytes through down-regulation of c-Jun N-terminal kinase. Rheumatology 53(9):1704-1711.

Lagathu C, Christodoulides C, Virtue S, Cawthorn WP, Franzin C, Kimber WA, Nora ED, Campbell M, Medina-Gomez G, Cheyette BN, et al. 2009. Dact1, a nutritionally regulated preadipocyte gene, controls adipogenesis by coordinating the Wnt/beta-catenin signaling network. Diabetes 58(3):609_ 619.

Lastra G, Manrique C. 2015. Perivascular adipose tissue, inflammation and insulin resistance: link to vascular dysfunction and cardiovascular disease. Horm Mol Biol Clin Investig 22(1):19-26.

Lin H, Angeli M, Chung KJ, Ejimadu C, Rosa AR, Lee T. 2016. sFRP2 activates Wnt/beta-catenin signaling in cardiac fibroblasts: differential roles in cell growth, energy metabolism, and extracellular matrix remodeling. Am J Physiol Cell Physiol 311(5):C710-C719.

Liu LB, Chen XD, Zhou XY, Zhu Q. 2018. The Wnt antagonist and secreted frizzled-related protein 5: implications on lipid metabolism, inflammation, and type 2 diabetes mellitus. Biosci Rep 38(4):BSR20180011.

Lu Y, Wang C, Hsu C, Chiu C, Yu T, Hung W, Lu L, Chung F, Tsai I, Lin H and others. 2013. Circulating secreted frizzledrelated protein 5 and wingless-type MMTV integration site family member 5 a levels in patients with type 2 diabetes mellitus. Diabetes Metab Res Rev 29(7):551-556.

Lv C, Jiang Y, Wang H, Chen B. 2012. Sfrp5 expression and secretion in adipocytes are up-regulated during differentia- 
tion and are negatively correlated with insulin resistance. Cell Biol Int 36(9):851-855.

Maekawa T, Kulwattanaporn P, Hosur K, Domon H, Oda M, Terao Y, Maeda T, Hajishengallis G. 2017. Differential expression and roles of secreted frizzled-related protein 5 and the wingless homolog Wnt5a in periodontitis. J Dent Res 96(5):571-577.

Malgor R, Bhatt PM, Connolly BA, Jacoby DL, Feldmann KJ, Silver MJ, Nakazawa M, McCall KD, Goetz DJ. 2014. Wnt5a, TLR2 and TLR4 are elevated in advanced human atherosclerotic lesions. Inflamm Res 63(4):277-285.

Maman E, Yung Y, Cohen B, Konopnicki S, Dal Canto M, Fadini R, Kanety H, Kedem A, Dor J, Hourvitz A. 2011. Expression and regulation of sFRP family members in human granulosa cells. Mol Hum Reprod 17(7):399-404.

Mastri M, Shah Z, Hsieh K, Wang X, Wooldridge B, Martin S, Suzuki G, Lee T. 2014. Secreted Frizzled-related protein 2 as a target in antifibrotic therapeutic intervention. Am J Physiol Cell Physiol 306(6):C531-C539.

Matsushima K, Suyama T, Takenaka C, Nishishita N, Ikeda K, Ikada Y, Sawa Y, Jakt LM, Mori H, Kawamata S. 2010. Secreted frizzled related protein 4 reduces fibrosis scar size and ameliorates cardiac function after ischemic injury. Tissue Eng Part A 16(11):3329-3341.

Mirotsou M, Zhang Z, Deb A, Zhang L, Gnecchi M, Noiseux N, Mu H, Pachori A, Dzau V. 2007. Secreted frizzled related protein 2 (Sfrp2) is the key Akt-mesenchymal stem cell-released paracrine factor mediating myocardial survival and repair. Proc Natl Acad Sci U S A 104(5):16431648.

Miyoshi T, Doi M, Usui S, Iwamoto M, Kajiya M, Takeda K, Nosaka K, Nakayama R, Okawa K, Takagi W, et al. 2014. Low serum level of secreted frizzled-related protein 5 , an anti-inflammatory adipokine, is associated with coronary artery disease. Atherosclerosis 233(2):454-459.

Mori H, Prestwich TC, Reid MA, Longo KA, Gerin I, Cawthorn WP, Susulic VS, Krishnan V, Greenfield A, Macdougald OA. 2012. Secreted frizzled-related protein 5 suppresses adipocyte mitochondrial metabolism through WNT inhibition. J Clin Invest 122(7):2405-2416.

Nakamura K, Sano S, Fuster JJ, Kikuchi R, Shimizu I, Ohshima K, Katanasaka Y, Ouchi N, Walsh K. 2016. Secreted frizzledrelated protein 5 diminishes cardiac inflammation and protects the heart from ischemia/reperfusion injury. J Biol Chem 291(6):2566-2575.

Okada Y, Sakaue H, Nagare T, Kasuga M. 2009. Diet-induced up-regulation of gene expression in adipocytes without changes in DNA methylation. Kobe J Med Sci 54(5):E241E249.

Ouchi N. 2011. [Obesity: progress in diagnosis and treatment; Topics, IV. Recent topics; 3. Obesity and new secretory factors; 2) Sfrp 5 acts as a novel adipocytokine that affects metabolic function]. Nihon Naika Gakkai Zasshi 100(4): 1002-1007.

Ouchi N, Higuchi A, Ohashi K, Oshima Y, Gokce N, Shibata R, Akasaki Y, Shimono A, Walsh K. 2010. Sfrp5 is an antiinflammatory adipokine that modulates metabolic dysfunction in obesity. Science 329(5990):454-457.

Patel VB, Shah S, Verma S, Oudit GY. 2017. Epicardial adipose tissue as a metabolic transducer: role in heart failure and coronary artery disease. Heart Fail Rev 22(6): 889-902.

Pawar NM, Rao P. 2018. Secreted frizzled related protein 4 (sFRP4) update: a brief review. Cell Signal 45:63-70.
Rebuffat SA, Oliveira JM, Altirriba J, Palau N, Garcia A, Esteban Y, Nadal B, Gomis R. 2013. Downregulation of Sfrp5 promotes beta cell proliferation during obesity in the rat. Diabetologia 56(11):2446-2455.

Rulifson IC, Karnik SK, Heiser PW, Ten BD, Chen H, Gu X, Taketo MM, Nusse R, Hebrok M, Kim SK. 2007. Wnt signaling regulates pancreatic beta cell proliferation. Proc Natl Acad Sci U S A 104(15):6247-6252.

Satoh W, Matsuyama M, Takemura H, Aizawa S, Shimono A. 2008. Sfrp1, Sfrp2, and Sfrp5 regulate the Wnt/ $\beta$-catenin and the planar cell polarity pathways during early trunk formation in mouse. Genesis 46(2):92-103.

Schulte DM, Kragelund D, Müller N, Hagen I, Elke G, Titz A, Schädler D, Schumacher J, Weiler N, Bewig B, et al. 2015. The wingless-related integration site-5a/secreted frizzledrelated protein-5 system is dysregulated in human sepsis. Clin Exp Immunol 180(1):90-97.

Schulte DM, Muller N, Neumann K, Oberhauser F, Faust M, Gudelhofer H, Brandt B, Krone W, Laudes M. 2012. Proinflammatory wnt5a and anti-inflammatory sFRP5 are differentially regulated by nutritional factors in obese human subjects. PLoS One 7(2): e32437.

Srivastava R, Zhang J, Go GW, Narayanan A, Nottoli TP, Mani A. 2015. Impaired LRP6-TCF7L2 activity enhances smooth muscle cell plasticity and causes coronary artery disease. Cell Rep 13(4):746-759.

Stuckenholz C, Lu L, Thakur PC, Choi TY, Shin D, Bahary N. 2013. Sfrp5 modulates both Wnt and BMP signaling and regulates gastrointestinal organogenesis [corrected] in the zebrafish, Danio rerio. PLoS One 8(4):e62470.

Suzuki H, Watkins DN, Jair KW, Schuebel KE, Markowitz SD, Chen WD, Pretlow TP, Yang B, Akiyama Y, Van Engeland M, et al. 2004. Epigenetic inactivation of SFRP genes allows constitutive WNT signaling in colorectal cancer. Nat Genet 36(4):417-422.

Takada I, Mihara M, Suzawa M, Ohtake F, Kobayashi S, Igarashi M, Youn MY, Takeyama K, Nakamura T, Mezaki Y, et al. 2007. A histone lysine methyltransferase activated by non-canonical Wnt signalling suppresses PPAR-gamma transactivation. Nat Cell Biol 9(11):1273-1285.

Tan X, Wang X, Chu H, Liu H, Yi X, Xiao Y. 2014. SFRP5 correlates with obesity and metabolic syndrome and increases after weight loss in children. Clin Endocrinol (Oxf) 81(3): 363-369.

Tang QQ, Lane MD. 2012. Adipogenesis: from stem cell to adipocyte. Annu Rev Biochem 81:715-736.

Teliewubai J, Bai B, Zhou Y, Lu Y, Yu S, Chi C, Li J, Blacher J, Xu Y, Zhang Y. 2018. Association of asymptomatic target organ damage with secreted frizzled related protein 5 in the elderly: the Northern Shanghai Study. Clin Interv Aging 13: 389-395.

Van Camp JK, Beckers S, Zegers D, Verhulst SL, Van Hoorenbeeck K, Massa G, Verrijken A, Desager KN, Van Gaal LF, Van Hul W. 2016. Nucleotide variation of sFRP5 gene is not associated with obesity in children and adolescents. Mol Biol Rep 43(10):1041-1047.

Vural B, Atalar F, Ciftci C, Demirkan A, Susleyici-Duman B, Gunay D, Akpinar B, Sagbas E, Ozbek U, Buyukdevrim AS. 2008. Presence of fatty-acid-binding protein 4 expression in human epicardial adipose tissue in metabolic syndrome. Cardiovasc Pathol 17(6):392-398.

Wang L, Wang Y, Meng Y, Zhang C, Di L. 2018. GSK3activated STAT5 regulates expression of SFRPs to modulate adipogenesis. FASEB J 32(9):4714-4726. 
Wang X, Peng Q, Jiang F, Xue L, Li J, Fan Z, Chen P, Chen G, Cai Y. 2017. Secreted frizzled-related protein 5 protects against oxidative stress-induced apoptosis in human aortic endothelial cells via downregulation of Bax. J Biochem Mol Toxicol 31(12):e21978.

Xu Q, Wang H, Li Y, Wang J, Lai Y, Gao L, Lei L, Yang G, Liao X, Fang X, et al. 2017. Plasma Sfrp5 levels correlate with determinants of the metabolic syndrome in Chinese adults. Diabetes Metab Res Rev 33(6):e2896.

Yin P, Wang W, Zhang Z, Bai Y, Gao J, Zhao C. 2018. Wnt signaling in human and mouse breast cancer: focusing on Wnt ligands, receptors and antagonists. Cancer Sci 109(11): 3368-3375.

Yu Y, Guan X, Nie L, Liu Y, He T, Xiong J, Xu X, Li Y, Yang $\mathrm{K}$, Wang Y, et al. 2017. DNA hypermethylation of sFRP5 contributes to indoxyl sulfate-induced renal fibrosis. J Mol Med (Berl) 95(6):601-613.

Zoico E, Darra E, Rizzatti V, Budui S, Franceschetti G, Mazzali G, Rossi AP, Fantin F, Menegazzi M, Cinti S, et al. 2016. Adipocytes WNT5a mediated dedifferentiation: a possible target in pancreatic cancer microenvironment. Oncotarget 7(15):20223-20235.

Zuriaga MA, Fuster JJ, Farb MG, MacLauchlan S, BretonRomero R, Karki S, Hess DT, Apovian CM, Hamburg NM, Gokce N, et al. 2017. Activation of non-canonical WNT signaling in human visceral adipose tissue contributes to local and systemic inflammation. Sci Rep 7(1): 17326 .

Address correspondence to:

Prof. Yujie Zhou

Department of Cardiology Beijing Anzhen Hospital Capital Medical University Beijing 100029

China

E-mail: azzyj12@163.com

Received 18 November 2018/Accepted 21 March 2019 